\title{
RECURSOS DIGITALES EN UNA HABITACIÓN DE ESCAPE EDUCATIVA
}

\section{DIGITAL TOOLS IN AN EDUCATIONAL ESCAPE ROOM}

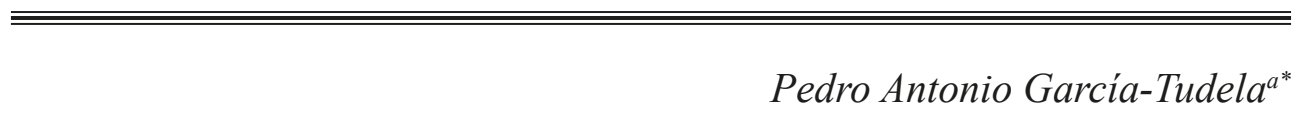

Fechas de recepción y aceptación: 10 de octubre de 2019, 8 de diciembre de 2019

Resumen: La sociedad digital actual plantea una serie de desafíos para cualquier contexto educativo. Por ello, en este trabajo se presenta una propuesta implementada en cuatro grupos de los grados de Educación Infantil y Primaria de la Universidad de Murcia para revisar contenidos relacionados con asignaturas de Tecnología Educativa antes de la prueba evaluativa final. La estrategia metodológica utilizada fue una habitación de escape educativa (Jumantic), la cual se basó tanto en recursos analógicos como digitales, ya que estos últimos son los menos utilizados en propuestas similares a la expuesta. La propuesta de innovación está basada en la película Jumanji, y a través de 9 puestos enigmáticos los estudiantes tenían que conseguir la llave para escapar del templo en el que se encontraban. Para resolver algunos de los enigmas tuvieron que utilizar robótica educativa a través de bee-bot, candados digitales, Kahoot, etc. Además, se desarrolló la práctica BYOD (Bring Your Own Device), para de esta forma escanear los diferentes códigos QR que se encontraban en el tablero de juego y marcar los diferentes itinerarios de los equipos. Igualmente, podían utilizarlos para consultar apuntes u otra información que considerasen de interés para resolver las diferentes preguntas relacionadas con el modelo TPACK, integración curricular de los medios, etc. A pesar de la negativa de ciertas propuestas a utilizar recursos digitales en las educational escape rooms. En este caso, los beneficios propios de la presente estrategia metodológica, se han visto

${ }^{a}$ Departamento de Didáctica y Organización Escolar. Facultad de Educación. Universidad de Murcia.

* Correspondencia: Universidad de Murcia. Facultad de Educación. Departamento de Didáctica y Organización Escolar. Campus Espinardo. 30100 Murcia. España.

E-mail: pedroantonio.garcia4@um.es 
complementados al incluir dicha visión digital. Por lo tanto, se considera de interés el hecho de diseñar, implementar e investigar propuestas similares como la descrita.

Palabras clave: habitación de escape educativa, tecnología educativa, recursos digitales, educación superior, grado en Educación Infantil, grado en Educación Primaria.

Abstract: Today's digital society poses a series of challenges for any educational context. Therefore, this paper offers a proposal implemented in four groups from the degrees in Preschool and Primary Education at the University of Murcia to study subjects related to Educational Technology before the final evaluation test. The methodological strategy used was an educational escape room (Jumantic), which was based on both analog and digital resources, since the latter are the least used in proposals similar to the one presented here. This innovation proposal is based on the movie Jumanji and through 9 enigmatic points students are expected to get the key to escape from the temple where they are located. To solve some of the riddles they had to use educational robotics through bee-bots, digital locks, Kahoot, etc. In addition, the BYOD (Bring Your Own Device) practice was also developed in order to scan the different QR codes that were on the game board and mark the different itineraries of the equipment. Likewise, they could use these to check notes or other information that they considered of interest to solve the different questions related to the TPACK model, curricular integration of the media, etc. This was carried out despite the refusal of certain proposals to use digital resources in educational escape rooms. In this case, the benefits of this methodological strategy have been complemented by including such digital vision. Therefore, it is considered of interest to design, implement and do research on similar proposals as described.

Keywords: educational escape room, educational technology, digital resources, higher education, degree in Pre-school Education, degree in Primary Education.

\section{MARCO TEÓRICO}

La educación superior, al igual que otros niveles educativos, se enfrenta a unos desafíos que parten de las demandas de su comunidad educativa. Uno de los retos más generalizados es progresar de un enfoque tradicional, en el que el alumnado posee un rol pasivo-asimilacionista, a uno en el que los estudiantes se involucren activamente en el proceso de enseñanza y aprendizaje, adquiriendo así un mayor compromiso y motivación (Adams, Burger, Crawford y Setter, 2018; Chi y Wylie, 2014).

A pesar de la negativa puntualizada sobre el uso de juegos en el contexto educativo formal (Blunt, 2007), se han evidenciado las oportunidades que estos pueden brindar. Con la implementación de estos, se puede estimular el 
pensamiento crítico sobre un tema, incrementar el interés por los contenidos trabajados, mejorar la motivación hacia el aprendizaje, facilitar la interacción con el entorno, etc. (Boyle, 2011; Strickland y Kaylor, 2016). Por esta razón, el aprendizaje basado en el juego (ABJ), es decir, la creación y/o implementación de juegos para trabajar unos contenidos y competencias (Pho y Dinscore, 2015), está siendo una de las líneas de investigación preferidas entre la comunidad científica correspondiente (Gallegos, Tesar, Connor y Martz, 2017).

Uno de los juegos más emergentes durante la última década, el cual se ha implantado en cualquier ciudad de alrededor del mundo, son las escape rooms o habitaciones de escape (Miller, 2015). Por su popularidad y su éxito entre la población joven (Gómez-Urquiza et al., 2019), se considera una oportunidad óptima para su inclusión como estrategia metodológica en la educación universitaria.

Concretamente, una sala de escape es un juego de tipo acción en vivo en el que un equipo, normalmente de 4 a 6 participantes o varios equipos, en caso de ser una sala de gran escala, han de escapar de un espacio cerrado. La manera de conseguirlo es resolviendo cooperativamente una serie de retos físicos y/o mentales que guardan relación con la narrativa de la sala (Connelly, Burbach, Kennedy y Walters, 2018; Novak, Lozos y Spear, 2019). Mayoritariamente, todas las escape rooms cuentan con una historia de trasfondo, la cual es la que plantea los objetivos y la situación inquietante por la que se ha de escapar del espacio ficticio (lugar del crimen, laboratorio, base militar, etc.) donde se encuentran los jugadores (Stasiak, 2016; Nicholson, 2015).

Tal y como ya se ha indicado, la mayoría de salas de escape son de uso exclusivo recreativo. No obstante, las educational escape rooms o escape rooms educativas demuestran el potencial que estas pueden brindar a cualquier nivel, como el fomento de la colaboración y el desarrollo de habilidades transversales como la socialización, entre otros beneficios propios del ABJ (Kinio, Dufresne, Brandys y Jetty, 2019).

De manera general, cuando se diseña una habitación de escape educativa se recomienda limitar el tiempo como en las originales (Nicholson, 2018). De esta forma se podrá tener una mayor gestión de la propuesta implementada, para así garantizar una óptima puesta en práctica. Además, también es favorable la reflexión de ciertos contenidos y de la experiencia en sí tras haber expirado el tiempo estimado como oportuno. 
En relación con el itinerario más recomendado, existen salas abiertas en las que independientemente del orden se tienen que resolver todos los enigmas para llegar al último; salas lineales, en la que el itinerario está preestablecido, o mixtas. Las más utilizadas son las segundas, ya que son menos desafiantes y la mejora del aprendizaje de unos contenidos dados puede ser más fácil (Connelly, Burbach, Kennedy y Walters, 2018; Nicholson, 2015). Asimismo, ayudará al docente a tener un mayor control de los equipos y del progreso del juego.

Por otro lado, se señala que además del tiempo y la planificación el último de los tres elementos esenciales son los recursos utilizados (Posey y Pintz, 2017). La gran mayoría de ellos suelen ser "analógicos", tales como puzles, carteles, cajas, candados, mensajes encriptados, etc., los cuales se afirma que tienen un precio mínimo (Choi, An, Shah y Singh, 2017; Eukel, Frenzel y Cermusca, 2017; Olszewsky y Wolbrink, 2017); sin embargo, en otros casos en los que se ha pretendido utilizar softwares u herramientas digitales se ha considerado que el coste es excesivo, y por dicha razón se han utilizado recursos como los descritos anteriormente (Kinio, Dufresne, Brandys y Jetty, 2019).

Tan solo se han utilizado vídeos de dibujos animados para informar sobre la narrativa de la habitación de escape educativa en un caso (Wise, Lowe, Hill, Barnett y Barton, 2018).

A pesar de la justificación de no utilizar recursos digitales por términos económicos, resulta paradójico que en plena era digital, en la que se demanda una educación digital (García-Aretio, 2019), no se utilice una muestra de estos. Sin embargo, existen bancos de dichos recursos y diversas posibilidades que permiten el uso de dispositivos tecnológicos sin coste alguno o mínimo (Córcoles, 2011; Doval y Raposo, 2014).

De la necesidad recién aludida parte el presente trabajo, cuyo objetivo es presentar una propuesta de innovación de una habitación de escape educativa, pero focalizado en los recursos digitales - sin coste- que se implementaron en ella. Debido a la discusión terminológica existente, es preciso especificar que se trata de una habitación de escape educativa real, no digital (Emre Dilek y Kulakoglu Dilek, 2018), es decir, un espacio físico enriquecido con recursos digitales. 


\section{Propuesta De INNOVACIÓN}

La propuesta didáctica basada en una escape room, a partir de la cual se especifican y analizan las diferentes herramientas digitales utilizadas durante su implementación, recibió el nombre de Jumantic'.

La habitación de escape educativa mencionada se diseñó exclusivamente para los estudiantes de las asignaturas relacionadas con la tecnología educativa de los grados de Educación Infantil y Primaria de la Universidad de Murcia. Concretamente, su objetivo era preparar al alumnado para el examen a través de un repaso de los principales contenidos, a la vez que se buscaba incrementar su motivación para la prueba evaluativa.

La temática de Jumanji favoreció la creación de un espacio enigmático a partir del que trabajar contenidos como el modelo TPACK, la utilización e integración curricular de los medios, entre otros.

Para ello, cada grupo que participó tuvo que crear 9 equipos, los cuales siguieron diferentes itinerarios dentro de la sala de escape. En esta, se encontraban 9 puestos enigmáticos, los cuales había que resolver sucesivamente para poder avanzar según la ruta marcada en el tablero principal de juego.

Cada grupo portaba consigo un diario de aventuras en el que anotaban la respuesta correcta de las 9 preguntas de múltiple opción que tuvieron que resolver. Más tarde, descubrieron que necesitaban todas las soluciones apuntadas por los diferentes equipos, ya que de otra forma sería imposible resolver el último enigma y escapar de la jungla.

Los diferentes enigmas que tenían que superar eran analógicos y digitales, y debido a la escasa presencia de los últimos en las habitaciones de escape educativas se considera de interés profundizar en estos para así favorecer el diseño de propuestas que contemplen herramientas digitales, las cuales son esenciales para educar en la sociedad digital actual (García-Novoa, 2019).

\footnotetext{
${ }^{1}$ Acceso al vídeo-resumen de la propuesta: http://bit.ly/2ntahdQ.
} 


\subsection{Herramientas digitales utilizadas en la propuesta de innovación}

Tal y como se ha mencionado, en el espacio donde se desarrolló la propuesta había una zona en la que se encontraba el tablero de juego (figura 1). Este recreaba el original de la película Jumanji, ya que había diferentes rutas, en este caso 9, cuya primera casilla tenía un color para diferenciar los diversos equipos.

En cada uno de los recuadros hasta llegar al centro se hallaba un código $\mathrm{QR}$, el cual debía ser escaneado por los miembros de cada color para saber a qué puesto enigmático debían dirigirse.

El orden que debían seguir con los códigos QR era sucesivo, ya que si alteraban el orden, dos equipos coincidirían para resolver el mismo enigma, lo cual es imposible, ya que existían 9 itinerarios diferentes para evitar este hecho.

Con el fin de garantizar un óptimo escaneado del tablero, se llevó a cabo una práctica de la tendencia extendida en las universidades, es decir, del Bring Your Own Device (BYOD) o "trae tu propio dispositivo" (Musarurwa, Gamundani y Bhunu Shava, 2019).

De manera previa a entrar en la sala de escape educativa, el alumnado tenía descargada la aplicación "QR code reader" en sus dispositivos móviles, para que de esta forma se optimizasen los tiempos.

Cada QR no facilitaba directamente el puesto enigmático al que se tenía que dirigir el alumnado, sino que ofrecía una pista a partir de la cual tuviesen que deducir cuál era el espacio correspondiente. Por ejemplo, la pista relacionada con el puesto analógico "escritorio del fotógrafo del rey" decía: "El fotógrafo del rey tiene que aplicar diferentes filtros para revelar sus fotografías. Visitad su escritorio de trabajo".

En relación con esta herramienta digital, también es preciso destacar que una vez que el game master tocaba una campana que siempre llevaba consigo los equipos debían regresar al tablero de juego para escanear el siguiente código QR. De esta manera, se tenía un control para evitar irregularidades, como escanear más de un código, y una mayor gestión temporal que favoreciese una práctica educativa efectiva. 
FIGURA 1

Tablero de juego de Jumantic

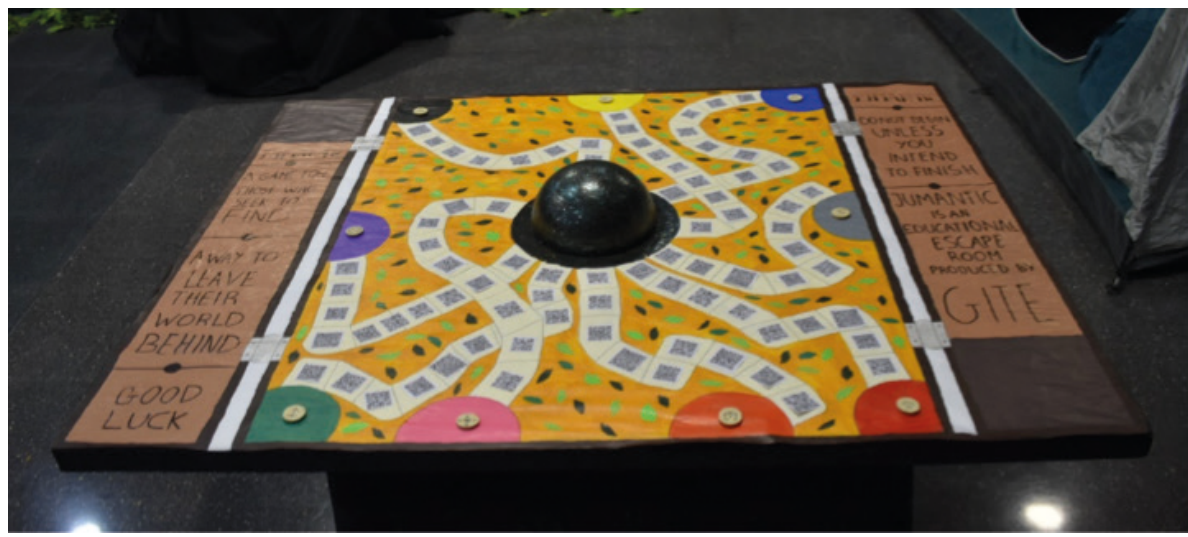

Fuente: elaboración propia

La gran mayoría de dispositivos móviles utilizados fueron smartphones, los cuales no solo se permitían para escanear códigos, sino que gracias a las posibilidades que el BYOD puede ofrecer al proceso de enseñanza-aprendizaje (Doval y Raposo, 2014) se consintió su utilización. Para ello, se estimó pertinente no crear una regla de juego que fuese no utilizar el móvil para resolver dudas, ya que también se quiso analizar la resolución de problemas en contextos como el descrito por parte del alumnado.

De esta manera, los estudiantes pudieron hacer uso de sus teléfonos móviles para consultar apuntes de la asignatura, la información que considerasen oportuna en páginas web, etc., y así resolver adecuadamente cada uno de los enigmas.

Tras estas dos herramientas digitales, es necesario aludir a la que se encontraba en el puesto "biblioteca del templo", es decir, un candado digital cargado en un ordenador portátil. Para desbloquear el dispositivo se debía resolver adecuadamente el enigma que aparecía en el QR pertinente ("Si el ordenador quieres desbloquear, la contraseña tendrás que acertar: Soy un número de 3 cifras. La suma de las tres cifras es 18 . La primera cifra es la mitad que la segunda y un tercio de la tercera ¿Qué número soy?’). 
Una vez resuelta la clave de acceso, aparecía un candado digital, es decir, una página web que plantea un desafío que se ha de resolver adecuadamente para acceder a cierta información. En este caso, un mensaje satisfactorio si se había resuelto adecuadamente o un aviso de que se tiene que volver a intentar.

El candado digital utilizado se puede consultar en el siguiente enlace: http:// bit.ly/30YZbLi. Una vez resuelto, los estudiantes anotaban la letra correcta en su diario de aventuras y esperaban a continuar con el siguiente código QR.

De manera muy relacionada con la herramienta digital explicitada, la que se encontraba en el puesto "despacho" era una tableta. Para llegar a ella, la pista escaneada mostraba: "Si la Tablet del despacho quieres desbloquear, la clave tienes que averiguar" y aparecía una imagen en la que se leía: “¿Qué letra falta aquí? LMM...VSD”. Una vez que dibujaban el patrón de la letra, se desbloqueaba y accedía a una cuestión en forma de Kahoot que tenían que responder.

La última herramienta digital empleada fue un robot educativo, ya que también es un contenido curricular de los grados participantes. Concretamente, se utilizó bee-bot, por la cercanía que tenían con su uso. La pista que los dirigía al puesto "zona de programación" fue: "A Ekmur le encanta programar su bee-bot. Seleccionad los últimos comandos que introdujo según vuestro color: http://bit.ly/30Wr5rf'.

Una vez dispuestos a resolver este enigma, encontraban una alfombrilla de cuadros, en algunos de los cuales había una tarjeta pegada. Por lo tanto, para descubrir cuál tenían que voltear para leer la cuestión que había debían programar el robot según el comando correspondiente a su color de equipo. Una vez que la bee-bot se posase sobre una tarjeta, esta era la que debían resolver.

Tal y como se ha expuesto, en esta experiencia de una habitación de escape educativa han compartido espacio recursos tanto analógicos como digitales. Específicamente, de los últimos se han empleado cinco diferentes, mientras que los puestos analógicos han sido seis. Por ello se establece un equilibrio significativo en cuanto a la diversidad de recursos utilizados. 


\section{CONCLUSiONES}

En el presente trabajo se ha dado a conocer una propuesta de innovación basada en una educational escape room, la cual se ha sustentado tanto en recursos digitales como en analógicos, a pesar de ser estos últimos los únicos utilizados en diversas prácticas educativas similares (Choi, An, Shah y Singh, 2017; Eukel, Frenzel y Cermusca, 2017, entre otros).

Si una habitación de escape educativa puede favorecer el alcance de diversos beneficios para el proceso de enseñanza y aprendizaje como su optimización, desarrollar habilidades fundamentales, facilitar la comunicación y la colaboración entre estudiantes y equipos, entre otros (Kinio, Dufresne, Brandys \& Jetty, 2019; Strickland y Kaylor, 2016), una propuesta que también contemple la inclusión de recursos digitales todavía brindará más oportunidades, ya que esta refleja de manera más completa la sociedad digital en la que vivimos (García-Novoa, 2019). Por lo tanto, el alumnado también podrá poner en práctica determinadas competencias digitales que se consideran fundamentales para su cotidianidad (Gomendio, 2019; Rodríguez, Raso y Ruiz, 2019). Además, sería paradójico implementar una educational escape room sobre contenidos de tecnología educativa en la que no hubiese recursos digitales.

De manera general, se justifica el empleo de puzles, cajas, candados, etc., por el excesivo coste de software y hardware. En este caso, independientemente de que ciertos recursos (bee-bot, tableta, etc.) son exclusivos del grupo de investigación en tecnología educativa de la Universidad de Murcia, existen diversas posibilidades con coste nulo o mínimo para desarrollar competencias digitales de manera paralela al desarrollo de una habitación de escape educativa (Musarurwa et al., 2019). Tan solo se necesitará uno o varios dispositivos tecnológicos y creatividad para valorar diferentes alternativas a la hora de diseñar los enigmas digitales.

A pesar de determinadas dificultades relacionadas con algunas cuestiones que se dieron en los diferentes grupos participantes, todos pudieron escapar en el límite de tiempo establecido, es decir, en 60 minutos. Pese al estrés y al rápido y efectivo ritmo de resolución de problemas, no se produjeron situaciones de desmotivación, de descontextualización del aspecto académico 
y focalización en la parte lúdica, no se ha superado el tiempo estimado como oportuno para su implementación, etc. (Pisabarro y Vivaracho, 2018).

Centrarse en los desafíos que la tecnología educativa puede ofrecer al contexto educativo. En esta propuesta tampoco se han producido grandes dificultades, puesto que en ningún momento han existido problemas de conexión de internet, roturas de recursos, etc. Tan solo en el caso de desbloquear con patrón o contraseña los dispositivos de la tableta y el ordenador portátil se produjeron errores que congelaron la pantalla por un tiempo. No obstante, este problema fue resuelto gracias a la interacción intergrupal, ya que debido al buen clima generado no se generó una competencia, sino todo lo contrario. Este hecho coincide plenamente con algunos hallazgos relacionados con buenas prácticas de tutoría entre iguales (Lara, 2001; Villanueva, 2001).

Asimismo, los resultados referentes a la temática trabajada, es decir, a la tecnología educativa, han sido también favorables. Algunas preguntas relacionadas sobre todo con el modelo TPACK, los aspectos relativos a la integración de los medios al contexto educativo y las funciones de estos han sido las que más dificultades presentaron al alumnado. Incluso, en algunos casos, hicieron uso del game master para plantearle las dudas que tenían al respecto. Sin embargo, de manera mayoritaria, las pudieron resolver posteriormente consultando los apuntes de la asignatura en sus smartphones.

Por último, este trabajo evidencia dos aspectos. Por un lado, las posibilidades que la educational escape room pueden brindar al progreso desde un enfoque únicamente tradicional basado en clases magistrales a otro en el que exista una amplia diversidad de estrategias metodológicas como la aquí presentada. Por otro lado, se ha demostrado la posibilidad de implementar recursos digitales en una habitación de escape educativa, ya que en algunos casos mencionados se considera un hecho imposible por la inversión económica requerida.

A partir de la información compartida, se recomienda la implementación e investigación de las potencialidades educativas que las salas de escape enriquecidas con TIC pueden tener en cualquier contexto formal. 


\section{BiBLIOGRAFÍA}

Adams, V., Burger, S., Crawford, K. y Setter, R. (2018). Can you escape? Creating an escape room to facilitate active learning. Journal for Nurses in Professional Development 34(2), E1-E5. doi: 10.1097/ NND.0000000000000433.

Blunt, R. (2007). Does games-based learning work? Results from three recent studies. Proceedings of the Interservice/industry Training, Simulation \& Education Conference. Orlando: National Defense Industrial Association (pp. 945-955). Recuperado de: http://bit.ly/2MnGCuU.

Boyle, S. (2011). Teaching Toolkit: and introduction to games based learning. Digital document. University College Dublin. Retrieved from: http:// bit.ly/32a63ak.

Chol, D., An, J., Shah, C. y Singh, V. (2017). Examining information search behaviors in small physical space: an escape room study. Proceedings of the Association for information Science and Technology 54(1). doi: https:// doi.org/10.1002/pra2.2017.14505401098.

Connelly, L., Burbach, B. E., Kennedy y C. Walters, L. (2018). Escape Room Recruitment Event: Description and Lessons Learned. Journal of Nursing Education 57(3), 184-187. doi: 10.3928/01484834-20180221-12.

Córcoles, J. E. (2011). Recursos Digitales para el aula. Quaderns digitals: revista de nuevas tecnologías y sociedad 67. Recuperado de: http://bit. ly/2B0rsXg.

Doval, M. I. y Raposo, M. (2014). Lecciones aprendidas de experiencias BYOD: Smartphones en la universidad, en J. Rodríguez y A. R. Fernández (eds.), Nuevas culturas y sus nuevas lecturas (pp. 157-167). Recuperado de: http://bit.ly/310ZBRs.

Emre Dilek, S. y Kulakoglu Dilek, N. (2018): Real-life escape rooms as a new recreational attraction: the case of Turkey. Anatolia 29(4), 495-506. doi: https://doi.org/10.1080/13032917.2018.1439760.

Eukel, H. N., Frenzel, J. E. y Cernusca, D. (2017). Educational Gaming for Pharmacy Students - Design and Evaluation of a Diabetes-themed Escape room. American Journal of Pharmaceutical Education 81(7). doi: 10.5688/ajpe 8176265 . 
Gallegos, C., Tesar, A. J., Connor, K. y Martz, K. (2017). The use of a game-based learning platform to engage nursing students: A descriptive, qualitative study. Nurse Educ. Pract. 27, 101-106. doi: 10.1016/j. nepr.2017.08.019.

GarcíA-Aretio, L. (2019). Necesidad de una educación digital en un mundo digital. RIED: Revista Iberoamericana de Educación a Distancia 22(2), 9-22. doi: http://dx.doi.org/10.5944/ried.22.2.23911.

García-NovoA, C. (ed.) (2019). $4 .{ }^{a}$ Revolución industrial: la fiscalidad de la sociedad digital y tecnológica en España y Latinoamérica. Madrid: Thomson Reuters Aranzadi.

Gomendio, M. (2019). Formar en competencias para un futuro digital. Executive excellence: la revista de liderazgo, la gestión y la toma de decisiones 154, 60-63.

Gómez-Urquiza, J., Gómez-Salgado, J., Albendín-García, L., Correa-Rodríguez, M., GonzÁlez-Jiménez, E. y Cañadas-De la Fuente. G. A. (2019). The impact on nursing students's opinions and motivation of using a "Nursing Escape Room" as a teaching game: a descriptive study. Nurse Education Today 72, 73-76. doi: https://doi.org/10.1016/j.nedt.2018.10.018.

Ho, A. M. (2018). Unlocking Ideas: Using Escape Room Puzzles in a Cryptography Classroom. Primus 28(9), 853-847. doi: https://doi.org/10.1080/ 10511970.2018.1453568.

Kinio, A.E., Dufresne, L., Brandys, T. y Jetty, P. (2019). Break Out of the classroom: the use of Escape Rooms as an Alternative Teaching Strategy in Surgical Education. Journal of surgical education 76(1), 134-139. doi: 10.1016/j.jsurg.2018.06.030.

LARA, S. (2001). Una estrategia eficaz para fomentar la cooperación. ESE: EStudios sobre educación 1, 99-110. Recuperado de: http://bit.ly/2Vt6U3e.

Miller, S. (2015). The art of the escape room. Recuperado de: http://bit. ly/2pbYIII.

Musarurwa, S., Gamundani, A. M. y Bhunu Shava, F. (2019). An assessment of BYOD control in higher learning institutions: a Namibian perspective, en IEEE (ed.), IST-Africa Week Conference. Kenya: Nairobi. doi: 10.23919/ISTAFRICA.2019.8764853.

Nicholson, S. (2015). Peeking behind the locked door: A survey of escape room facilities. Recuperado de: http://bit.ly/351Kp4J. 
Nicholson, S. (2018). Creating Engaging Escape Rooms for the Classroom. Childhood Education 94(1), 44-49. doi: https://doi.org/10.1080/00094056 .2018.1420363.

Novak, J., Lozos, J. C. y Spear, S. E. (2019). Development of an Interactive Escape Room Intervention to Educate College Students about Earthquake Preparedness. Natural Hazards Review 20(1). doi: 10.1061/(ASCE) NH.1527-6996.0000322.

Chi, M. T. H. y Wylie, R. (2014). The ICAP framework: linking cognitive engagement to active learning outcomes. Educ. Psychol 49, 219-243. doi: https://doi.org/10.1080/00461520.2014.965823.

Olszewski, A. E. y Wolbrink, T. A. (2017). Serious gaming in medical education: a proposed structured framework for game development. Simulation in Healthcare 12(4), 240-253. doi: https://doi.org/10.1097/ SIH.0000000000000212 .

Pho, A. y Discore, A. (2015). Game-Based Learning. Tips and trends. Instructional Technologies Committee. Recuperado de: http://bit.ly/33hdmwQ.

Pisabarro, A. M. y Vivaracho, C. E. (2018). Gamificación en el aula: gincana de programación. ReVisión: Revista de Investigación en Docencia Universitaria de la Informática 11(1), 85-93. Recuperado de: http://bit. ly/2pduiFD.

Posey, L. y Pintz, C. (2017). Original research: transitioning a bachelor of science in nursing program to blended learning: successes, challenges \& outcomes. Nurse Education in Practice 26, 126-133. doi: 10.1016/j. nepr.2016.10.006.

Rodríguez, A. M., Raso, F. y Ruiz, J. (2019). Competencia digital, educación superior y formación del profesorado: un studio de meta-análisis en la web of science. Pixel-Bit: Revista de medios y educación 54, 65-82. doi: https://doi.org/10.12795/pixelbit.2019.i54.04.

STASIAK, A. (2016). Escape rooms: A new offer in the recreation sector in Poland. Tourism 26(1), 31-47. doi: 10.1515/tour-2016-0003.

Strickland, H. P. y KaIlor, S. K. (2016). Bringing your A-game: Educational gaming for student success. Nursing Education Today 40, 101-103. doi: 10.1016/jnedt.2016.02.014. 
VillanueVA, L. (2001). El aprendizaje cooperativo en historia: diseño de actividades y efectos cognitivos y sociales (tesis doctoral). Murcia: Universidad de Murcia. Recuperado de: http://bit.ly/2q2HfCQ.

Wise, H., Lowe, J., Hill, A., Barnett, L. y Barton, C. (2018). Escape the welcome cliché: Designing educational escape rooms to enhance students' learning experience. Journal of Information Literacy 12(1), 86-96. doi: http:// dx.doi.org/10.11645/12.1.2394. 